\title{
El discurso neoliberal y la configuración de la identidad profesional en docentes noveles
}

The neoliberal discourse and the configuration of professional identity in novice teachers

\section{Noralí Boulan ${ }^{1}$}

\section{Resumen}

Este artículo estudia los sentidos otorgados a la educación en la provincia de Buenos Aires durante la gestión de gobierno de María Eugenia Vidal (2015-2019). La hipótesis de trabajo supone que la política desplegada por el gobierno provincial rearticuló el discurso neoliberal menemista, aunque sumando nuevos elementos. Las ideas relativas a la educación emocional y las capacidades, asociaron la calidad educativa a la evaluación de resultados y la productividad, conformándose en los significantes que articularon la interpelación oficial a la docencia. De acuerdo con ello, se describen los ejes de la actualización curricular para el nivel primario y su recepción por parte de los docentes noveles, analizando cómo los posicionamientos que conforman su identidad profesional aceptan o disputan dichos sentidos.

Palabras clave: Discursos; identidad docente; currículum.

\section{Abstract}

This article studies the meanings given to education in the province of Buenos Aires during the administration of María Eugenia Vidal (2015-2019). The working hypothesis assumes that the policy deployed by the provincial government

\footnotetext{
${ }^{1}$ Tutora del Programa de Doctorado en Estudios Latinoamericanos de la Universidad Nacional de La Plata (UNLP, Argentina). Becaria Posdoctoral del Consejo Nacional de Investigaciones Científicas y Técnicas (CONICET, Argentina). Email: noraliboulan@gmail.com
} 
rearticulated the menemist neoliberal discourse, although adding new elements. The ideas related to emotional education and capabilities, associated educational quality to the evaluation of results and productivity, conforming in the signifiers that articulated the official interpellation to teaching. Accordingly, we describe the axes of the curricular update for the primary level and its reception by novice teachers, analyzing how the positionings that shape their professional identity accept or dispute these meanings.

Key words: Discourses; teaching identity; curriculum.

\section{Introducción}

El trabajo forma parte de una investigación en curso, cuyo objetivo es realizar un análisis epistemológico de algunas categorías introducidas por la actualización del diseño curricular para la Educación Primaria de la provincia de Buenos Aires en 2018, instaurada bajo la gestión neoliberal del gobierno de Mauricio Macri en Argentina y, asimismo, la gestión de dicha provincia, a cargo de la gobernadora María E. Vidal.

El artículo se basa en la investigación doctoral ${ }^{2}$ que describió y analizó las identificaciones construidas desde la política curricular bonaerense entre los años 2006 y 2016, donde la búsqueda en torno a un "posicionamiento docente" resultaba el eje de la formación del profesorado. Uno de los propósitos del presente trabajo es estudiar el proceso de construcción de la identidad profesional analizando la interpelación que el nuevo discurso educativo propone a los y las docentes ${ }^{3}$ en el momento de su inserción laboral. Comprendemos, de este modo, que allí tensionan diversas interpelaciones, pero también diferentes recepciones de los sentidos de las prescripciones estatales en dos direcciones. En primer lugar, resulta necesario considerar que han sido formados sobre la base de la política educativa que, instaurada luego de la implementación de la Ley de Educación Nacional No 26.2006

\footnotetext{
2 Boulan, N. (2019). Políticas de formación de profesores de nivel primario en la provincia de Buenos Aires. Discursos, curriculum y procesos identificatorios (2006-2016) [Tesis de Doctorado]. Universidad Nacional de La Plata. Argentina.

${ }^{3}$ En el texto se utilizará el genérico masculino solo con objeto de facilitar la lectura, pese a que los datos estadísticos y de campo demuestran como característica particular una mayoritaria participación femenina en el campo profesional docente.
} 
[LEN, 2006], los interpeló en su formación donde la noción de criticidad retomada desde la pedagogía freireana se suponía fundamental dentro del rol profesional, con objeto de formar un "posicionamiento docente". En segundo lugar, se intenta indagar, las herramientas por ellos utilizadas para desarrollar su trabajo bajo la prescripción curricular para el Nivel Primario de la Provincia de Buenos Aires, que posee características distintas a las líneas didácticas con las cuales han sido formados e introduce categorías tales como "capacidades" y "desarrollo emocional" y donde la evaluación de resultados y la eficacia de los mismos cobran suma importancia tanto a nivel nacional como provincial.

Myriam Southwell (2015) utiliza la categoría "posición docente" para dar cuenta de los procesos, nunca predeterminados ni fijados, donde los docentes disputan y reformulan los sentidos de las políticas públicas. Esta noción permite comprender la identidad docente como una construcción y, en tanto tal, los sentidos que asume son siempre abiertos e inacabados. Desde este lugar, en este artículo analizamos el discurso educativo neoliberal configurado bajo la gestión macrista, tanto a nivel nacional como provincial, por entender que allí existió una disputa de sentidos a la identidad docente pero donde existen tensiones dentro del propio campo curricular, aún configurado en un discurso hegemónico. Consideramos necesario reformular la pregunta por la identidad constituida desde la política curricular y pensar, antes, cómo se desarrolla la misma, entendiendo que siempre existe allí una disputa por su sentido.

\section{Metodología}

La investigación supone un trabajo teórico-conceptual. Se incluye en el trabajo de campo entrevistas a docentes noveles del nivel primario y docentes de los profesorados de tres regiones educativas ${ }^{4}$ de la Provincia de Buenos Aires. Éstas

\footnotetext{
${ }^{4}$ La Provincia de Buenos Aires se organiza en veinticinco regiones educativas que agrupan a los 135 municipios que la componen. Las regiones educativas son concebidas como la instancia de conducción, planeamiento y administración de la política educativa. Según el artículo 58 de Ley de Educación de la Provincia $N^{\circ} 13.688$, cada Región Educativa comprende a uno o más de un distrito conforme a los componentes comunes que los agrupen.
} 
fueron seleccionadas por la diversidad contextual, económica y social de la provincia, a fin de obtener una muestra representativa.

La opción metodológica se concentró en el Análisis Político del Discurso [APD], como perspectiva analítica posfundacional y no-esencialista, retomando el análisis de los textos primarios de referentes del enfoque teórico, tales como Ernesto Laclau y Chantal Mouffe (2004), Rosa Nidia Buenfil Burgos (2010), Myriam Southwell (2015), Alicia de Alba (1995), relativos a las nociones de "identidad", "posiciones docentes" e "interpelación". Se procedió al relevamiento y recopilación de fuentes documentales relativas a la política educativa a nivel nacional y, específicamente, los documentos curriculares para la educación primaria de Buenos Aires, y de las circulares y comunicaciones elaboradas a propósito de dicha implementación. Estas tareas permitieron reconstruir el marco más amplio de las políticas educativas en las que se insertan las propuestas curriculares para la educación primaria desarrollada en la provincia.

Las entrevistas a docentes noveles que han vivido la transición entre diseños curriculares, que deben trabajar con el nuevo Diseño Curricular para la educación primaria en el momento de su inserción profesional, tuvieron como objeto indagar si existen allí tensiones entre la formación que han recibido y las concepciones que propone la prescripción oficial con la cual deben trabajar, entre las cuales se encuentran las categorías "capacidades" y "educación emocional".

En este sentido, resulta necesario señalar, en primer lugar, que la formación docente para el nivel primario en la provincia de Buenos Aires se encuentra a cargo de los Institutos Superiores de Formación Docente [ISFD]. Asimismo, en esta investigación se define como docentes noveles a aquellos graduados recientes de dichas instituciones considerando un lapso menor a cinco años. De acuerdo con ello, uno de los criterios de selección de los participantes fue considerar el tiempo transcurrido desde el momento de la entrevista y la fecha de egreso de la institución formadora. Dado que las entrevistas se desarrollaron durante el año 2020, un principio excluyente fue que los sujetos participantes hubieran egresado del profesorado a partir del año 2016. En segundo lugar, un dato a considerar en la selección de las participantes es el género, ya que una de las particularidades de la 
docencia en Argentina, así como en Latinoamérica, es su carácter femenino. La feminización de la docencia ha sido una temática ampliamente estudiada desde la historia de la educación y los datos muestran que en la actualidad esa característica permanece. Según el último Censo Nacional del Personal de los Establecimientos Educativos [CENPE], realizado en el país en 2014, del total de docentes en actividad en la educación común -que incluye los niveles inicial, primario y secundario- el 76, $5 \%$ son mujeres, el $21,9 \%$ son varones, sin registrarse datos en el $1,6 \%$ restante (CENPE, 2014).

De acuerdo con lo anterior, se realizaron nueve entrevistas semi estructuradas a docentes noveles para obtener información que permitiera realizar un análisis comparativo y denso respecto de algunos aspectos considerados centrales. De este modo, se entrevistó a tres docentes de la región educativa 1, que comprende entre sus distritos a la capital provincial, cuatro docentes la región educativa 4, compuesta por distritos pertenecientes a la zona sur del Gran Buenos Aires, y dos docentes de la región educativa 11, comprendida por cinco distritos de la zona noreste de la provincia, compuestos por zonas urbanas y semi - rurales $)^{5}$.

Respecto del rango etario, la mayoría de las entrevistadas se encuentra entre los 23 y 30 años de edad. Sólo en uno caso, la entrevistada revela una edad mayor a los 30 años. Asimismo, todas las participantes se formaron en instituciones de gestión estatal y poseen una antigüedad en el trabajo docente que oscila entre uno y cuatro años. En algunos casos, además de la experiencia situada dentro de la formación inicial en el proceso de residencias en las escuelas primarias, las experiencias laborales se originaron durante el proceso de formación docente inicial6. Las entrevistas realizadas a docentes noveles se organizaron a partir de tres grandes ejes que permitieran conocer:

\footnotetext{
${ }^{5}$ De los cinco partidos que componen la región educativa 11, dos de ellos forman parte de los 30 partidos que el Instituto Nacional de Estadística y Censos [INDEC] denomina "partidos del aglomerado Gran Buenos Aires" pero no pertenecen a los 24 partidos que el INDEC denomina "partidos del Gran Buenos Aires" en sentido administrativo.

${ }^{6}$ En la provincia de Buenos Aires el ingreso a la docencia dentro del sistema educativo formal puede desarrollarse a partir de la aprobación del $50 \%$ de las materias que constituyen el profesorado. En líneas generales estas primeras experiencias ocurren paralelamente a la formación, bajo el formato de suplencias de docentes con cargos titulares.
} 
- Las trayectorias formativas: año de graduación del profesorado, antigüedad en la docencia en el nivel primario, formación continua -cursos, seminarios transitados luego del egreso de la formación inicial-.

- La situación en la cual se encontraban al producirse la actualización curricular para el nivel primario -durante su formación en el profesorado, en los primeros años de inserción laboral-.

- La valoración que realizaban sobre la correlación y/o discontinuidad entre los enfoques pedagógicos recibidos durante la formación propuesta por el plan de estudios de 2007, y los presentados por la actualización curricular para el nivel primario (2018).

Por otro lado, también se realizaron entrevistas a dos docentes de la asignatura "Campo de la Práctica Docente" del profesorado. La decisión de su incorporación en el trabajo de campo fue relativa a que se trata de los docentes a cargo de las residencias ${ }^{7}$ que realizan los estudiantes del profesorado en las escuelas asociadas. El objetivo, en estos casos, se relacionó con obtener información relevante respecto del proceso de actualización curricular en el nivel primario (2018) y cómo ello repercutió en la formación docente, cuyo diseño curricular, implementado desde el año 2007 se orienta por enfoques pedagógicos y didácticos críticos, que no resultaban concordantes con los nuevos sentidos que organizan algunos ejes de la política curricular.

En todos los casos, las entrevistas se realizaron mediante el consentimiento informado de los participantes estableciendo la garantía de anonimato. (Mainardes y Carvalho, 2019). Los discursos de las personas participantes se codificaron del siguiente modo: docente de la región educativa 4 con cuatro años de antigüedad en la docencia (Docente novel 1), docente de la región educativa 11 con dos años de antigüedad en la docencia (Docente novel 2), docente de la región educativa 1 con tres años de antigüedad en la docencia (Docente novel 3), docente de la región

\footnotetext{
7 En los últimos dos años de la formación docente inicial, los estudiantes del profesorado realizan sus residencias en escuelas primarias. Las mismas se llevan adelante dentro de la materia "Campo de la práctica docente" y se encuentran a cargo no sólo de los docentes titulares de dicha asignatura, sino que resultan acompañadas por los docentes a cargo del curso en el cual se efectiviza la residencia.
} 
educativa 4 con tres años de antigüedad en la docencia (Docente novel 4) y dos docentes del profesorado de la región educativa 4 (Docente del profesorado 1) y (Docente del profesorado 2).

Los resultados parciales de la investigación permiten explorar la hipótesis según la cual, los docentes noveles han interiorizado durante su formación inicial posicionamientos basados en pedagogías críticas. Ello produce que posean lecturas complejas de las actualizaciones de las políticas curriculares $y$, además, un distanciamiento de las nociones respecto de la educación emocional y la evaluación de productos procedentes del discurso educativo neoliberal evidenciado en el diseño curricular con el que deben organizar sus propuestas de enseñanza.

La investigación propuesta es exploratoria, dado que existe un número reducido de estudios sobre el tema. El abordaje metodológico es principalmente cualitativo, en tanto se procura generar conocimiento a partir de los datos empíricos del trabajo de campo. Se produce así un proceso en espiral de ida y vuelta constante entre obtención de datos y generación de teoría, a través del cual se procura focalizar en los aspectos más sustantivos del problema de investigación planteado (Glaser y Strauss, 1967; Sautú, 2003; Sirvent, 2005; Taylor y Bogdan, 1986).

\section{El discurso neoliberal y la rearticulación de los sentidos acerca de lo "educativo"}

En 2015 en Argentina se produce el ascenso al poder del gobierno de Mauricio Macri (2015-2019) luego de tres periodos de gobiernos kirchneristas: la presidencia de Néstor Kirchner (2003-2007) y los dos periodos de gobierno de Cristina Fernández de Kirchner (2007-2011; 2011-2015), que tuvieron características netamente diferentes respecto de las políticas públicas y modelos de gestión del Estado.

Durante las gestiones de gobierno del kirchnerismo se establecieron diversas políticas que supusieron la ampliación de derechos en vastos sectores sociales. De este modo es posible afirmar que la categoría "inclusión" fue el significante vacío 8

\footnotetext{
${ }^{8}$ Con el uso de la categoría significante vacío se hace referencia a ciertos términos que son objeto de una lucha ideológica muy fuerte en la sociedad; por ello, estos términos van a propender a ser significantes tendencialmente vacíos -nunca totalmente vacíos- por el hecho de que dada la pluralidad de conflictos que ocurren alrededor de ellos no pueden ser fijados a una única
} 
que resultó capaz de articular diversas y muy dispares demandas sociales (Southwell y Vassiliades, 2016). Ello repercutió en el ámbito educativo en general, y en un movimiento circular, en la formación docente, reestructurando sus sentidos, siendo capaz de rearticular viejas y nuevas demandas en la arena de disputa por determinar lo social (Laclau y Mouffe, 2004). En dicha configuración discursiva la educación cobró un sentido fundamental, considerada como un derecho individual y social. Además, estrictamente en el ámbito pedagógico, la noción de transmisión se asoció a las vertientes críticas respecto de la educación como vía de transformación social. La "centralidad de la enseñanza" se vio así relacionada a la posibilidad de construcción de escenarios más igualitarios (Vassiliades, 2012). La idea de igualdad, por su parte, se tornó equivalente de la de inclusión y se emparentó a la principalidad estatal, la afirmación del derecho social a la educación y la restitución de lo común. Eso se reflejó, fundamentalmente, en el tratamiento de las diversidades, la promoción de otras modalidades educativas, la implementación de la Asignación Universal por Hijo, el establecimiento del carácter nacional de la educación y del piso común de aprendizajes para el estudiantado.

En trabajos anteriores (Boulan, 2019; Southwell y Boulan, 2019) hemos analizado cómo en la Provincia de Buenos Aires, en el año 2007 se estableció el nuevo Diseño Curricular para la Formación Docente que se estructuró bajo el eje de la praxis. Comprendiendo la politicidad del acto educativo, interpeló a la docencia desde un paradigma pedagógico crítico, asumiendo la construcción de un "posicionamiento docente" autónomo, situado y contextuado, en el marco de la comprensión de la educación como derecho social. Allí describíamos la reformulación curricular como un proceso no lineal, donde fue posible observar las resistencias que los docentes establecían al respecto. Un proceso de negociación permanente entre la interpelación oficial y los sujetos destinatarios de dicha política.

articulación discursiva. Y justamente, en el hecho de las disputas que suscitan en torno a ellos, reside su potencialidad y capacidad de interpelación. Aquí conviene detenerse en una confusión habitual, porque suele entenderse rápidamente que el calificativo de vacío habla en desmedro del término; muy por el contrario, el vaciamiento parcial habla de la potencia de un significante que logra absorber a otros, se vacía parcialmente al mismo tiempo que incorpora sentidos y significaciones que lo exceden (Laclau, 1996). 
Comprender los planes de estudio como configuradores de subjetividades y como campo de disputa por sus significantes implica, en términos de Alicia de Alba (1995), a todos los sujetos del desarrollo curricular. Ello procura, por tanto, comprender a los docentes no sólo como sujetos destinatarios de determinadas políticas sino como constructores de las mismas. Emprender ese camino supuso una habilitación y el reconocimiento de su poder de decisión. De este modo, la consulta previa a la reformulación del diseño curricular para la formación docente impulsada por la gestión de gobierno provincial entre los años 2004 y 2007 se configuró como un proceso de retroalimentación entre los "sujetos de determinación curricular", los "sujetos de estructuración formal del curriculum" y los "sujetos de desarrollo curricular", con una orientación que apeló a la construcción participativa y con cierto nivel de horizontalidad en la toma de decisiones.

Por otro lado, el macrismo conformó su discurso no sólo por oposición al gobierno antecesor, sino que, sin perder totalmente sus sentidos, los disputó y rearticuló. La concepción macrista del Estado, asociada al gerenciamiento del mismo, unida a la afirmación de la ineficacia de la burocracia estatal funcionaron como elementos que cohesionaron un discurso donde la función de la educación se planteó como bastión de la transformación social para "insertar a la Argentina en el mundo". En efecto, el slogan de campaña electoral: "Sí, se puede" y el propio nombre de la alianza que llevara al gobierno al presidente Mauricio Macri, "Alianza Cambiemos", aglutinaron dispares sentidos, cuyo significante articulador fue el "cambio".

En esta dirección, Paul Du Gay (1996) señala cómo la crítica a la organización burocrática supone que la idea de cambio cultural es un elemento constitutivo del discurso gerencial contemporáneo y añade que el contexto organizacional parece confirmar que toda cuestión relativa a la cultura remite a cuestiones de identidad. De acuerdo con esto, se considera que aquellos que posean las normas, valores y actitudes correctas, es decir, quienes hayan realizado un cambio cultural, se convertirán en "hombres de negocios" exitosos. El autor afirma que el lenguaje del "cambio" es un elemento constitutivo del discurso empresarial $y$, al generarse en forma relacional, excluye otras acepciones, concluyendo que el discurso gerencial se opone al discurso burocrático. El diagnóstico de los distintos aspectos sociales, 
económicos, educativos, culturales y políticos realizados por el macrismo al inicio de su gestión fue plasmado en diversos documentos oficiales y la conformación de la retórica discursiva del "cambio" ligada a una lógica exitista, fue plasmada por diversos funcionarios de gobierno en declaraciones públicas.

De acuerdo con lo anterior, el análisis de las principales políticas educativas promulgadas por el gobierno permite afirmar que la "revolución educativa" se transformó en uno de los significantes centrales del discurso educativo, que, aunque articulada con la noción de "inclusión" también condensó diversos sentidos que procuraron asociar la evaluación de productos con la calidad educativa. De igual modo, la crítica respecto de la organización burocrática e ineficiente del Estado estuvo acompañada por un continuo desprestigio al trabajo y a la formación docente, mediante declaraciones públicas de diversos funcionarios de gobierno tal como la remitida por el ministro de educación y deportes, Esteban Bullrich, quien afirmó que el Plan Estratégico 2016-2021 "Argentina Enseña y Aprende", creado mediante la resolución 285/16 del Consejo Federal de Educación [CFE], se asemejaba a "una nueva campaña del Desierto [...]" $]^{\prime \prime}$. Asimismo, el propio jefe de estado en marzo de 2017, en medio del conflicto con los sindicatos docentes por la reducción del presupuesto educativo y el reclamo por la reapertura de las negociaciones paritarias, al presentar los resultados del Operativo Aprender $(2016)^{10}$, responsabilizó de los malos resultados al estado de la educación pública, dando lugar a una campaña de desprestigio de la misma: "Es increíble que cinco de cada diez chicos no comprendan un texto en la escuela pública. En la escuela privada, son dos de cada diez. $Y$ en eso también tenemos que trabajar, en terminar con la terrible inequidad entre aquel que puede ir a una privada y aquel que tiene que caer en la escuela pública"11.

\footnotetext{
9 Declaración pública del Ministro de Educación y Deportes transmitida por diversos medios masivos de comunicación, en la inauguración del nuevo Hospital Escuela de Veterinaria dependiente de la Universidad Nacional de Río Negro. 11 de septiembre de 2016.

10 Evaluación estandarizada nacional que se encuentra en sintonía con las evaluaciones internacionales tales como las propuestas por el Programme for International Student Assessment [PISA].

${ }^{11}$ Declaración pública del presidente Mauricio Macri, difundida en diversos medios masivos de comunicación, en el marco de la conferencia de prensa para la presentación de los resultados del operativo de evaluación nacional "Aprender". 21 de marzo de 2017.
} 
En el contexto bonaerense, la gestión de la gobernadora María E. Vidal (20152019) impulsó un discurso concordante con el de la gestión de la política nacional en el ámbito educativo. A través de programas de capacitación tales como el Proyecto "Escuelas en red" y la reformulación curricular para la educación primaria, se rearticularon sentidos tecnocráticos, neoliberales y mercantilistas. Mediante la lectura exhaustiva de documentos elaborados por la nueva gestión de gobierno, tanto en el ámbito nacional como en el provincial, expresiones públicas de funcionarios de los mismos, así como la revisión de fuentes secundarias, fue posible observar cómo el discurso gerencial y nociones tales como el "emprendedurismo" se orientaron a la reconfiguración del significante "inclusión" a partir de ideas ligadas a la eficiencia y la meritocracia, al tiempo que en un movimiento circular, asociaron los resultados de las evaluaciones internacionales a la calidad educativa, en un intento de desplazamiento de la noción de la educación como "derecho social" impulsada desde la Ley de Educación Nacional (2006).

\subsection{La "revolución educativa" bonaerense y los sentidos de la actualización curricular}

En consonancia con los sentidos otorgados a la educación por el gobierno nacional, en la Provincia de Buenos Aires, bajo la gestión de María E. Vidal desde 2015, se recupera la significante calidad como eje del discurso educativo. Alejandro Finocchiaro, ministro de educación bonaerense (2015-2017), apeló en declaraciones públicas a la "educación del futuro", "la revolución educativa" y la incorporación de las nuevas tecnologías para mejorarla e insertar a los alumnos en un mundo incierto, además de señalar la importancia de la formación docente para lograr dicha meta. De acuerdo con ello, en una entrevista otorgada a la Revista "El arcón de Clio" en 2016 el funcionario definía los conceptos centrales de lo que consideraba una "revolución de la educación", asociándola a la calidad educativa:

La educación de calidad es la educación que impacta produciendo en los alumnos aprendizajes significativos [...] que los inserte en el siglo XXI. Hoy nosotros tenemos escuelas del siglo XIX con docentes del siglo XX con alumnos del siglo XXI, hay que educar para el nuevo siglo. Un chico que tiene 
hoy entre diez y doce años es posible que cambie siete u ocho veces de trabajo en el transcurso de su vida, cinco de esos trabajos todavía no fueron creados, entonces nosotros [...] tenemos que incorporar todas las tecnologías nuevas para que el alumno esté inserto. (párrafo 3)

Asimismo, en cuanto a las desigualdades sociales y el rol de la educación en su disminución, apela a la calidad educativa, pero disociada del componente político transformador que había caracterizado el discurso educativo de la gestión anterior. Así, se relaciona "calidad" con "talento" y "meritocracia", al tiempo que recupera los sentidos otorgados en los Contenidos Básicos Comunes $(\mathrm{CBC})^{12}$ que se desplegaron durante el gobierno neoliberal menemista (1989-1999), entendiendo como "saberes significativos" las aptitudes, conocimientos y valores. De este modo afirma:

[...] el objetivo es en primer lugar igualar el punto de partida, hoy si uno nace en un lugar geográfico tiene mejores posibilidades de que le vaya bien en la vida que si nació en otros lugares geográficos. [...] y eso lo logra la educación, por eso lo que tenemos que hacer, nuestra gran responsabilidad, es empezar a construir una educación de calidad en la provincia de Buenos Aires, [...] la que produce saberes significativos. Es decir, aquel conjunto de valores, conocimientos y aptitudes que van hacer que el día de mañana cada uno de nuestros alumnos pueda llegar en la vida hasta donde su capacidad, su esfuerzo y su motivación le dicte, pero para eso nosotros tenemos que prepararlos. (2016, párrafo 4)

En relación con ello, el ministro refuerza la idea de la necesidad de capacitación y formación docente a partir de un diagnóstico donde la recopilación de información resulta central. De este modo, la calidad educativa se asocia a la innovación y la incorporación de las nuevas tecnologías de la comunicación.

Vamos a brindar capacitación a los maestros de la Provincia. Estará enfocada en las buenas prácticas, en cómo y cuál es la mejor manera de enseñar y en las nuevas formas de aprender de los alumnos del siglo XXI. [...] Por eso, la capacitación se enfocará también en el uso pedagógico e inteligente de las

\footnotetext{
${ }^{12}$ En la política curricular de los años noventa, los Contenidos Básicos Comunes para el Nivel Inicial y la Educación General Básica agrupaban el conjunto de saberes a enseñar dentro de tres ejes: los "contenidos conceptuales/disciplinares", los "contenidos procedimentales" y los "contenidos actitudinales".
} 
nuevas tecnologías, indispensables para el desarrollo docente y el trabajo de la escuela. [...] Tenemos que trabajar sobre las prácticas docentes en el aula, sobre capacitación y formación docente. (2016, párrafo 4)

Desde esta perspectiva, la gestión provincial anunció en 2016 el programa "Buenos Aires hace escuela", propuesta de la Dirección de Formación Continua "[...] destinada a crear instancias de formación innovadoras que profundicen y estimulen el trabajo de los equipos de las escuelas bonaerenses" (p.2). De acuerdo con esto, el programa se basa en dos ejes centrales: "alumnos protagonistas" y "el papel de las nuevas tecnologías". Según Guillermina Tiramonti (2016), directora de la cartera a cargo del mismo, se buscaba la innovación de las prácticas áulicas mediante la capacitación, comprendiendo a los docentes como "conductores de propuestas". Realizando un diagnóstico comparativo con experiencias anteriores, la funcionaria afirma que la propuesta supone proveerle a los profesores "[...] una idea de cómo puede trabajar distinto y de cuáles son los instrumentos que necesita para hacerlo." (p. 6). Según el subsecretario de educación bonaerense, Sergio Siciliano, "[...] la propuesta busca concentrar la formación docente en las prácticas áulicas, fundamentalmente con cuatro grandes líneas de trabajo: prácticas del lenguaje, matemática y operaciones lógicas y la incorporación de tecnologías dentro de las escuelas" (2016, p. 3).

Como puede observarse, existe una noción de calidad educativa ligada a un diagnóstico que supone que la implementación de propuestas formativas donde los docentes son interpelados como sujetos destinatarios de propuestas predefinidas por la prescripción de las políticas educativas y programas a implementar. Esto se aleja de la noción de posicionamiento que procuraba articular la propuesta curricular del Profesorado de Nivel Primario (2007). Situar a los docentes como "conductores de propuestas" implica a la vez, posicionarlos en un rol de simples aplicadores de la prescripción, es decir, como "sujetos de desarrollo curricular" (De Alba, 1995).

En 2017, la cartera educativa bonaerense fue ocupada por el economista Gabriel Sánchez Zinny, quien reemplazó a Alejandro Finocchiaro, nombrado como ministro de educación y deportes en el ámbito nacional. Durante la gestión de Sánchez Zinny (2017-2019) se produjeron actualizaciones curriculares para todos los 
niveles educativos: inicial, primaria, secundaria, educación técnica profesional y algunos profesorados para el nivel secundario. No obstante, la propuesta formativa para los Profesorados de Nivel Inicial y Primario, promulgada en 2007 no se modificó. Esta situación generó que se interrumpiese cierta correlación entre las propuestas con las cuales se formaba a los docentes del nivel primario y los planes de estudio diseñados para el nivel en que deben llevar adelante sus prácticas pedagógicas.

En efecto, tanto la actualización de Diseño Curricular para el Nivel Inicial y Primario (Resolución No 1482/17) como el Marco Referencial Curricular (Resolución No 4358/18) afirman que los mismos fueron producto de la consulta y del trabajo mancomunado con diversos actores sociales y educativos, tal como se planteó la legislación curricular anterior, con objeto de "avanzar en un recorrido hacia la consolidación de una propuesta aún más enriquecida, actualizada y de calidad para la formación de los alumnos del Nivel Primario de la Provincia de Buenos Aires" (Resolución No 1482/17, p. 6). En ese sentido, las normas mencionadas sugieren un trabajo que continúa la línea de las gestiones anteriores, sosteniendo que durante los años 2016 y 2017 se realizó la evaluación de los diseños curriculares y se implementaron acciones que procuraban "identificar fortalezas y debilidades en la organización y abordaje de los contenidos curriculares de los niveles". De igual modo, se señalaque se efectuaron "asistencias técnicas, encuentros de trabajo con inspectores, directores y docentes, encuestas territoriales y resultados de operativos de evaluación" y que la información relevada "se constituyó en un eje fundamental que orientó la toma de decisiones para la revisión y actualización de los diseños curriculares" (Resolución N॰4358/18, p. 2).

Consultadas por la participación en el proceso de actualización curricular, ninguna de las docentes entrevistadas dio cuenta del mismo. Sólo en un caso, se menciona la recepción de un "documento preliminar" en la institución, que fue trabajado en una jornada institucional. (Docente novel 1). En el resto de los casos, las entrevistadas afirman desconocer el proceso de consulta, informándose sobre la actualización curricular sólo en el momento de su implementación. 
Asimismo, el Diseño Curricular para el Nivel Primario (Resolución $\mathrm{N}$ ○ 1482/17), presenta como innovaciones dos módulos: "Educación Inclusiva" y el "Módulo TIC" considerados ejes transversales al resto de las áreas curriculares. En ambos casos, su contenido no difiere de los contenidos ya presentes en la normativa anterior. No obstante, en las áreas curriculares correspondientes a la enseñanza de las prácticas del lenguaje, las matemáticas y las ciencias, los sentidos tecnocráticos se evidencian más solventemente, lo cual tuvo una fuerte repercusión en las reflexiones realizadas por las docentes noveles entrevistadas:

Notamos una diferencia importante en el área de Prácticas del Lenguaje que en el diseño del 2008 era muy constructivista y aparece este nuevo diseño volviendo a hablar de la conciencia fonética. Eran cambios muy pequeños que podían pasar desapercibidos, pero había que estar atentos. Otro cambio importante fue en Ciencias Sociales y Ciencias Naturales, hubo reducción de caja horaria. Nosotros teníamos tres módulos semanales de cada ciencia y lo bajaron a dos y yo pienso que las ciencias son igual de importantes como lo son Matemáticas y Prácticas del Lenguaje. Desde mi punto de vista, no me parecía tan bueno quitarles espacio a esas dos áreas. También lo que nos llamó un poco la atención es que en este nuevo diseño aparece el módulo inclusión. Nos hizo como un poco de ruido, porque no es algo nuevo, lo planteaban como un avance, una gran propuesta y la verdad que en las escuelas es algo cotidiano y algo que se usó toda la vida, no es una innovación. Eso sonó un poco raro, pero, en general, no noté grandes diferencias y grandes cambios. Lo que sí noté es que quizás no hay tanto apoyo teórico en este nuevo diseño como sí lo había en el anterior, el del 2008. (Docente novel 1)

Cuando se hizo el cambio de diseño, yo estaba en $4^{\circ}$ año de la carrera y las profesoras de las didácticas nos comentaban la manera en que se cambiaron los contenidos, recuerdo que estaban muy enojadas por los recortes de contenidos, especialmente la profesora de Naturales. (Docente novel 2) 
Cuando leí la propuesta había algo que no me cerraba en lo que era la formación que tuve de cuatro años en el Instituto. Hablo de las Prácticas del Lenguaje, encontraba esta diferencia de abordaje o enfoque pedagógico en torno a las Prácticas del Lenguaje que realmente no lo pude hacer, la verdad que es como ir en contra de mis principios. (Docente novel 3)

Nosotros siempre seguimos el lineamiento de la escuela liberadora de Freire. Cuesta, porque venimos de una escuela tradicional y, a veces, uno en su práctica misma salió un poco el conductismo, pero la realidad es que siempre nos posicionamos de este lado, en el recorrido pedagógico del profesorado que fue nuevo y nos resultó revolucionario, de intentar enseñar por este camino. (Docente novel 4)

Por otro lado, la noción de calidad educativa se asocia a la responsabilidad individual en cuyo caso cada sujeto es constructor de su futuro en función de sus triunfos, fracasos, su "mérito" independientemente de las condiciones contextuales en las que se inserte. Así, el Marco Curricular Referencial (Resolución N • 4358/18) de la provincia señala la "necesidad de definir perfiles de egreso por desarrollo de capacidades", en un extenso apartado donde explicita

[...] Se entiende por capacidades la combinación de saberes, habilidades, valores y disposiciones. Las capacidades cognitivas, emocionales y sociales están inextricablemente entrelazadas a lo largo de toda la vida. [...] Una educación que fomenta habilidades cognitivas juntamente con habilidades conductuales, tales como la atención, el autocontrol, la motivación y la sociabilidad, convierten el conocimiento en experiencia que atraviesan transversalmente los contenidos disciplinares y las áreas de conocimiento (Res. 330/17 CFE). Las capacidades fundamentales establecidas en el Marco Orientador de los Aprendizajes (MOA), se relacionan con las competencias de educación digital, que promueven la alfabetización en el área para una inserción plena de los estudiantes en la cultura contemporánea y en la sociedad. Estas capacidades son coincidentes con lo señalado en los conceptos que dan fundamento a la Red Federal de 
Educación, para la mejora de los aprendizajes. A su vez, en el marco del Plan Estratégico Nacional (Res.285/16 - CFE) el documento "Marco Nacional de Integración de los Aprendizajes: hacia el desarrollo de capacidades", presenta el desarrollo de las capacidades como foco de la organización curricular de la enseñanza, a los fines de favorecer el proceso de los aprendizajes de calidad que sostengan trayectorias educativas integrales y continuas [...] (Resolución $\mathrm{N} \circ 4358 / 18$, pp. 25-26)

Lo anterior permite mostrar cómo la categoría "capacidades" se asocia ineludiblemente a la gestión de las emociones y a la incorporación de las nuevas tecnologías de la información y la comunicación [NTICs]. Asimismo, se relaciona estrechamente a las NTICs a la "calidad" de los aprendizajes de los estudiantes.

\subsection{El Proyecto "Red de escuelas de aprendizaje" y la educación como mercancía. Entre saludos, recreos cerebrales y emoticones}

En el marco del proceso de actualización curricular, basado en el nuevo módulo de "Educación Inclusiva" del Diseño Curricular para la Educación Primaria (2018) según el cual "se incluyen estrategias y herramientas para el aprendizaje basado en proyectos disciplinarios e interdisciplinarios por ciclo [que] serán elaborados por la Dirección Provincial de Educación Primaria y se ofrecerán como materiales complementarios para la enseñanza y el aprendizaje" (p. 17), la Dirección Provincial de Evaluación y Planeamiento puso en marcha el Proyecto "Red de Escuelas de Aprendizaje" entre los años 2017 y 2019. Su presentación explicita que el propósito es "[...] que los miembros de las comunidades educativas que la componen aprendan entre pares, reflexionen colaborativamente, intercambien buenas prácticas y se motiven entre sí para lograr una mejora escolar continua generada por actitudes positivas en los directivos, docentes y estudiantes." (2019, p. 9). Según el informe final, del programa participaron más de 2.000 escuelas de modo voluntario.

En líneas generales, el funcionamiento del programa replica el formato de diversos proyectos internacionales tales como Teach For All. En efecto, Agustina 
Blanco, directora de la propuesta, forma parte del consejo asesor de "Enseña por Argentina", la versión local del programa internacional.

De acuerdo con el informe, la Red ofreció capacitación a docentes para que se transformaran en los docentes referentes, denominados especialistas, a la vez que cumplían con el rol de ser multiplicadores de las propuestas en sus instituciones. Las capacitaciones abarcaron las Prácticas del Lenguaje, Matemática, Clima Escolar y Educación Emocional, Aprendizaje Basado en Proyectos (ABP), Fortalecimiento de Alfabetización y Acompañamiento de Trayectorias Escolares. Sin embargo, una de las áreas que cobró mayor relevancia fue "Clima Escolar y Educación Emocional" cuyo sustento teórico se funda en la psicología de Daniel Goleman y el desarrollo de la inteligencia emocional. Al respecto y consultada sobre la participación en el proceso de diseño de la actualización curricular para el Nivel Primario de la Provincia de Buenos Aires, una docente del Campo de la Práctica del Profesorado de Educación Primaria señala:

No fui convocada para ninguna consulta ni me enteré que los docentes de escuela primaria o equipos directivos fueran convocados por parte del ministerio. Sí sé que fueron convocadas algunas escuelas, para el proyecto de "Red de Escuelas de Aprendizaje", a una capacitación que dirigía la Dirección Provincial del Planeamiento, que, podríamos decir, se encontraba en línea con el nuevo sentido que intentaron darle al nuevo diseño curricular para la educación primaria: la educación emocional y el trabajo de aprendizaje por proyecto. Lo que observamos de la propuesta "Red de Escuelas" era una simplificación de la enseñanza emocional reducida a emoticones o a la presencia o no de saludos. (Docente del profesorado 1)

Tal como señala la entrevistada, las capacitaciones docentes en el marco de la reformulación curricular para el Nivel Primario fueron llevadas a cabo por la Dirección de Planeamiento y Evaluación del gobierno bonaerense. En línea con los sentidos que articularon el discurso educativo nacional, el anexo de la Resolución que establece el marco curricular plantea como "punto de partida" la información producida por las pruebas estandarizadas "PISA", así como su versión nacional, la evaluación "Aprender" a partir del cual, se responsabiliza fuertemente de esta situación al sistema de formación docente: 
Un aspecto muy importante, en términos de generar procesos formativos de calidad, es la propia característica del sistema formador. Se han iniciado procesos y definido diversas estrategias para lograr una mayor focalización en las problemáticas y situaciones que suceden en la realidad de las escuelas. Según el Instituto Nacional de Formación Docente, las propuestas pedagógicas de las instituciones con ofertas de capacitación responden parcialmente a las necesidades de los alumnos del siglo XXI, señala asimismo, que aún subsisten prácticas tradicionales de enseñanza, escaso uso de las nuevas tecnologías y dificultad en la secuenciación de los contenidos, todos aspectos que responden a una escasa actualización pedagógica y curricular acorde a los nuevos formatos y modelos de enseñanza en función de las exigencias de la sociedad actual. En consecuencia, se torna indispensable la intervención pedagógica y la actualización de la formación docente inicial, así como también la de los docentes que se desempeñan actualmente en el sistema educativo bonaerense. (Resolución $N^{\circ} 4358 / 18$, p. 3)

Lo anterior se refleja en el testimonio de otra de las docentes de un profesorado entrevistada respecto de las capacitaciones del proyecto bonaerense "Red de escuelas de aprendizaje"

Participé en uno de esos encuentros de capacitación del proyecto. En febrero de 2018 me convocaron desde la Dirección de Evaluación, porque iban a trabajar con los resultados del Operativo Aprender. Cuando presentaron los resultados de la evaluación, armaron diez equipos de trabajo con diez animadores. Cuando se presentaron notamos que había una variedad de profesionales: enfermeros, médicos, administradores, pero ninguno era profesor o pedagogo, lo cual nos resultaba extraño. Al acercarse el animador al equipo nuestro, le pregunto: ¿ustedes son del Ministerio de Nación, son del equipo de Evaluación de Nación? No, era una empresa contratada, obviamente era una organización no gubernamental contratada para llevar adelante esta capacitación, el gobierno nacional y provincial contrataba empresas en vez de utilizar los equipos técnicos de los ministerios. (Docente del profesorado 2)

En relación a ello, recuperamos los planteos de Stephen Ball (2014), quien supone la existencia de una "nueva economía de la educación" asociada con 
diversos modos de privatización de los servicios educativos, así como las modificaciones en las formas y modalidades del Estado. Según el autor, en el contexto mundial se están produciendo nuevos tipos de negocios educativos en donde la educación se convierte en una mercancía que genera ganancias a partir de la inserción en el ámbito educativo de inversiones mediante Alianzas PúblicoPrivadas (APP) e Iniciativas de Financiamiento Privado (PFI). Entre estos negocios o edupreneurs existen distintos servicios, entre los cuales se hallan programas educativos, capacitación profesional y desarrollos curriculares que legitiman un nuevo discurso educativo promovido por organizaciones y grupos de interés que movilizan procesos de reforma pública. Así, señala que "La OCDE también proporciona un andamiaje discursivo para la privatización de los servicios públicos a través de la noción de 'contestabilidad', término que se refiere a la incorporación de proveedores alternativos de servicios públicos (estatales, privados, comunitarios, etc.)" (pp. 4-5).

Al mismo tiempo, remarca la existencia de las famosas thinks tanks que promueven la privatización educativa a través de inversiones y la generación de un nuevo marco regulatorio en el ámbito del Acuerdo General de Comercio en Servicios (AGCS) que reglamenta los flujos internacionales de servicios educativos privados. De acuerdo con ello, el AGCS determina que una vez que un servicio público es suministrado por un proveedor no estatal, aunque sea nacional, no puede negarse entonces la entrada de proveedores internacionales. En este sentido es interesante destacar que en los ejemplos incorporados en su análisis el autor menciona el caso argentino como un país en el cual se produce dicha situación. Según Ball, la existencia de "flujos internaciones" supone la "tercerización de la educación", a la que caracteriza siguiendo a Patrinos (2005) como

[...] un proceso por el cual un gobierno proporciona servicios educativos o relacionados con la educación por un volumen y cantidad definidos, a un precio acordado, de un proveedor específico y por un período de tiempo delimitado. El acuerdo entre el proveedor y quien financia el servicio queda establecido en un contrato. (p. 5) 
De acuerdo con lo anterior, sostiene que se produce la importación de un "nuevo modelo de gestión", donde la tercerización es la meta de esta nueva forma de inversión en los mercados internacionales. A su vez, afirma que otro campo de los nuevos negocios educativos es el de la "exportación y venta de políticas públicas" (p. 9). De este modo, se "venden" reformas educativas, programas de evaluación y políticas educativas como "productos enlatados", sin considerar los contextos particulares de aplicación de las mismas.

En el caso de la provincia de Buenos Aires dicha tercerización se produjo por la sustitución progresiva de los sistemas de capacitación docente que gratuitamente otorgaba el Estado a partir de la introducción de contratos con Organizaciones No Gubernamentales, tanto nacionales como internacionales. De acuerdo con ello, mediante la Resolución No 733 del año 2018 la Dirección General de Cultura y Educación firmó un convenio con la Fundación Varkey. No obstante, la presencia de dichas ONGs no es un caso aislado de la provincia, sino que se trata de una alianza público-privada bajo un convenio entre el Ministerio de Educación Nacional, la Fundación Varkey y la fundación de origen nacional "Educere", cuya fundadora es Victoria Zorraquín, quien ocupó la Dirección de Formación Docente Continua de la Provincia de Buenos Aires en la gestión de la gobernadora Vidal.

Tal como señala Ball (2007), las nociones de productividad en ámbito educativo se encuentran asociadas al establecimiento de rankings determinados, entre otros, por las pruebas estandarizadas. En el caso bonaerense, las actualizaciones, reformas y capacitaciones docentes estuvieron fundamentadas en el bajo desempeño de los estudiantes en las pruebas "Aprender". Siguiendo los planteos del autor se convierte a la educación en un proceso de producción, reenfocando las actividades en base a los resultados, pero fundamentalmente, habilita el pensamiento educativo en función de su productividad. Asimismo, la introducción de la lógica empresarial modifica la metodología utilizada por los profesores, quienes conciben el aula como un laboratorio capaz de maximizar resultados, a partir de procedimientos memorísticos y repetitivos en sus estudiantes.

En este sentido, la propuesta de educación emocional fue ampliamente criticada por diversos sectores del ámbito educativo, sindicatos docentes e incluso, 
los docentes destinatarios alzaron rápidamente la voz, y denunciaron que ésta encubría la mercantilización de la educación pública. En efecto, respecto de una de las jornadas de capacitación, el Sindicato Único de los Trabajadores de la Educación de la Provincia de Buenos Aires (SUTEBA) expresó en una nota en su sitio web:

El Gobierno Provincial, a través de la Dirección de Formación Continua, convocó a los Equipos de Formación a una jornada con la presencia de la Fundación Varkey. En esta jornada quedó explícito que se sigue desmantelando el Sistema Formador Público y que intentan mercantilizar la Formación para impactar en la Escuela con las lógicas del mercado. Rutinas de respiración y control emocional, música relajante para tranquilizar la conciencia y conectarse con el yo interior, "recreos cerebrales" para alivianar tensiones... Son sólo algunas de las políticas educativas que impulsa la gestión de Victoria Zorraquín de Pereyra Iraola para la Formación continua de Ixs Docentes. (SUTEBA, 2019, s/p.) ${ }^{13}$

Mediante los diversos análisis realizados, podemos afirmar que el discurso educativo neoliberal tanto en las gestiones nacional como provincial supuso una nueva configuración que planteaba el programa "Escuelas del futuro", a partir de cadenas equivalenciales formadas por conceptos tales como "innovación", "calidad", "modernización", "inserción en el mundo", "cultura digital", "mérito", "liderazgo" y "calidad". En la misma dirección, la propuesta de "educación inclusiva", presentada como una innovación en la provincia de Buenos Aires, procuró desplazar el sentido político de la misma, conformando un configuración discursiva que emparentó la "gestión de las emociones" con el coaching emocional y, además, reorientó los planes de estudio de modo tal que la interpelación a la docencia se fusionó con ideas de profesores como conductores de propuestas prediseñadas, simples ejecutores y/o multiplicadores de programas evaluados como exitosos. Dichas caracterizaciones pueden señalarse como el intento de desligar de todo sentido "político" a las prácticas educativas, entendiendo a la educación como mercancía y como "vehículo para insertarse en el mundo".

${ }^{13}$ El uso del lenguaje inclusivo se encuentra en la versión original. 


\section{Conclusiones}

Comenzamos este artículo describiendo brevemente el modo en que el modelo de estado neoliberal bajo las gestiones nacional y provincial de la Alianza Cambiemos se asoció con una gestión eficiente de los recursos y se conformó por oposición a la burocracia estatal, ligada a sentidos negativos respecto de las políticas implantadas por la gestión de gobierno anterior. Ello permitió analizar el contexto en el cual resulta posible señalar la reconfiguración del discurso educativo como el marco más amplio de las disputas por sus sentidos, es decir, en el marco de lo social y lo político (Laclau, 1996). Bajo este mismo mecanismo, la nueva configuración retoma elementos de discursos anteriores, pero lo realiza al tiempo que incorpora nuevos significantes que logran una rearticulación de sus sentidos. De este modo, la educación, bajo la gestión de gobierno neoliberal, si bien continuó formulándose ligada a los significantes "derecho" e "inclusión", se asoció a las categorías eficiencia y eficacia, en pos de lograr "la incorporación de la Argentina al mundo".

Asimismo, el diagnóstico realizado respecto de la formación docente en el ámbito bonaerense, y el consecuente e inevitable camino hacia su "mejoramiento", se presentó desde programas verticalistas, basados en directrices de ejecutivos empresariales. Se permitió la injerencia de "los nuevos negocios educativos" (Ball, 2014), al incorporar ONGs nacionales e internacionales en las políticas educativas y curriculares, tercerizando la educación como una nueva forma de privatización. Por último, dicha reconfiguración del discurso se produjo por dos vías paralelas: el "diagnóstico" de la mala calidad del sistema educativo y la consecuente importancia otorgada a la evaluación, cuyos modelos se proponen desde los organismos internacionales, sin considerar los procesos, contextos y posicionamientos diversos en los cuales se desarrollan las prácticas de enseñanza.

Así, la "revolución educativa", fue el significante que procuró articular los sentidos "evaluación", "innovación" y "capacitación". Ello permitió estructurar un discurso que interpeló a los docentes desde un lugar técnico; "facilitadores", "conductores de prácticas educativas que permiten a los alumnos insertarse en un 
mundo cambiante", "veedores" en el desempeño de los estudiantes en la aplicación de evaluaciones estandarizadas.

No obstante, a partir del testimonio de las entrevistadas, es factible observar cómo surgen distintas recepciones de las políticas educativas que son rearticuladas por otros significantes, mostrando un posicionamiento muy crítico a las nociones propuestas por el discurso provincial. Ello permite inferir que dichos sentidos no son asumidos acríticamente, sino que, por el contrario, existe una fuerte disputa por los mismos.

Tal como señalan diversos autores, las interpelaciones resultan una "invitación" a ser, pero nunca logran completar totalmente la falta (Buenfil Burgos, 2010; Laclau y Mouffe, 2004). Para resultar exitosas necesitan que los sujetos interpelados se reconozcan allí donde están siendo nombrados, aunque esa investidura nunca es completa, absoluta, esencial, en tanto se disputa en terreno de lo indecidible (Southwell, 2015).

\section{Referencias bibliográficas}

Ball, S. (2007). Education plc: Understanding private sector participation in public sector education. London: Routledge.

Ball, S. (2014). Globalización, mercantilización y privatización: tendencias internacionales en Educación y Política Educativa. Archivos Analíticos de Políticas Educativas, 22(41). http://dx.doi.org/10.14507/epaa.v22n41.2014

Boulan, N. (2019). Políticas de formación de profesores de nivel primario en la provincia de Buenos Aires. Discursos, curriculum y procesos identificatorios (2006-2016) [Tesis de Doctorado] Universidad Nacional de La Plata. Argentina. Disponible en https://bit.ly/33tYMEt

Buenfil Burgos, R. (2010). Dimensiones ético políticas en educación desde el análisis político de discurso. Sinéctica, Revista Electrónica de Educación, 35, 1-17.

De Alba, A. (1995). Curriculum: crisis, mito y perspectivas. Buenos Aires: Miño y Dávila. 
Dirección General de Cultura y Educación (2016). Programa "Buenos Aires hace escuela". Disponible en https://bit.ly/3f1P3dY

Dirección General de Cultura y Educación (2019). Red de Escuelas de Aprendizaje. Informe 2019. Disponible en https://bit.ly/3vR95yx

Du Gay, P. (1996). Organización de la identidad: gobierno empresarial y gestión pública. En S. Hall y P. Du Guy (Comp.), Cuestiones de identidad cultural (pp.251-280). Madrid: Amorrortu Editores.

Finocchiaro, A. (2016). Hay que educar para el nuevo siglo. Entrevistado por Daniela Seisdedos. Revista -El arcón de Clio. Disponible en https://bit.ly/33sxbmU

Glaser, B. y Strauss, A. (1967). The discovery of grounded theory. Chicago: Aldine.

Laclau, E. y Mouffe, C. (2004). Hegemonía y estrategia socialista. Buenos Aires: Fondo de Cultura Económica.

Laclau, E. (1996). Emancipación y diferencia. Buenos Aires: Ariel.

Mainardes, J. y Carvalho, I. (2019). Autodeclaração de princípios e procedimentos éticos. En Associação Nacional de Pós-Graduação e Pesquisa em Educação. Ética e pesquisa em Educação (pp. 129-132). Rio de Janeiro. ANPED.

Ministerio de Educación Nacional (2014). Censo Nacional del Personal de los Establecimientos Educativos. Disponible en https://bit.ly/33qizEH

Resolución No 4358/18 "Marco Curricular Referencial", Dirección General de Cultura y Educación, La Plata, 20 de noviembre de 2018.

Resolución No 1482/17 "Diseño curricular para la educación primaria: primer ciclo y segundo ciclo", Dirección General de Cultura y Educación de la Provincia de Buenos Aires, 15 de noviembre de 2017.

Sautú, R. (2003). Todo es teoría. Objetivos y métodos de investigación. Buenos Aires: Lumiere.

Sirvent M. (2005). El Proceso de Investigación. Buenos Aires: Cuadernos de la Oficina de Publicaciones de la Facultad de Filosofía y Letras (Opfyl).

Southwell, M. (diciembre de 2015). La construcción de posiciones docentes frente a situaciones de desigualdad social y educativa. Ponencia presentada en el 
seminario Figuras actuales de la Segregación FLACSO, Área de Educación. Buenos Aires.

Southwell, M. y Vassiliades, A. (2016). Articulaciones y disputas en la trama entre trabajo docente e igualdad educativa: una aproximación histórica. En G. Brenner y G. Galli (Comp.), Inclusión y calidad como políticas educativas de Estado o el mérito como opción única de mercado (pp. 33-52). Buenos Aires: Stella.

Southwell, M. y Boulan, N. (2019). Política de formación docente en la provincia de Buenos Aires (2004-2007): Curriculum, horizontes formativos y praxis política. Revista Estado y Políticas Públicas, 13, 61-82.

Sindicato Único de Trabajadores de la Educación de la Provincia de Buenos Aires (2019). "Disciplinar las emociones, educar para el mercado". Disponible en https://bit.ly/3xYAwbm

Vassiliades, A. (2012). Regulaciones del trabajo de enseñar en la provincia de Buenos Aires: posiciones docentes frente a la desigualdad social y educativa. [Tesis de Doctorado] Universidad de Buenos Aires. Argentina.

Taylor, S. y Bogdan, R. (1986) Introducción a los métodos cualitativos de investigación. Buenos Aires: Paidós. 